\title{
Chondrogenic Priming at Reduced Cell Density Enhances Cartilage Adhesion of Equine Allogeneic MSCs - a Loading Sensitive Phenomenon in an Organ Culture Study with 180 Explants
}

\author{
Jan H. Spaas ${ }^{a}$ Sarah Y. Broeckx ${ }^{a}$ Koen Chiers ${ }^{b}$ Stephen J. Ferguson c,d \\ Marco Casarosac Nathalie Van Bruaene ${ }^{a}$ Ramses Forsyth ${ }^{e}$ Luc Duchateau ${ }^{f}$ \\ Alfredo Franco-Obregón ${ }^{g, h, i}$ Karin Wuertz-Kozak ${ }^{c, d}$ \\ ${ }^{a}$ Global Stem cell Technology, ANACURA group, Evergem, Belgium; 'Department of Pathology, \\ Bacteriology and Poultry Diseases, Faculty of Veterinary Medicine, Ghent University, Merelbeke, \\ Belgium; 'Institute for Biomechanics, ETH Zurich, Zurich, Switzerland; ${ }^{\mathrm{d} C o m p e t e n c e ~ C e n t e r ~ f o r ~ A p p l i e d ~}$ \\ Biotechnology and Molecular Medicine CABMM, University of Zurich, Zurich, Switzerland; 'Department \\ for Anatomo-pathology, Brussels University Hospital, Brussels, Belgium; 'Department of Comparative \\ Physiology and Biometrics, Faculty of Veterinary Medicine, Ghent University, Merelbeke, Belgium; \\ פDepartment of Surgery, Yong Loo Lin School of Medicine, National University of Singapore, Singapore; \\ hDepartment of Physiology, National University of Singapore, Singapore; 'National University Hospital \\ Sports Centre, National University of Singapore, Singapore
}

\section{Key Words}

MSCs $•$ Cartilage $\cdot$ Chondrogenic $\cdot$ Horse $\cdot$ Peripheral Blood

\begin{abstract}
Background: Clinical results of regenerative treatments for osteoarthritis are becoming increasingly significant. However, several questions remain unanswered concerning mesenchymal stem cell (MSC) adhesion and incorporation into cartilage. Methods: To this end, peripheral blood (PB) MSCs were chondrogenically induced and/or stimulated with pulsed electromagnetic fields (PEMFs) for a brief period of time just sufficient to prime differentiation. In an organ culture study, PKH26 labelled MSCs were added at two different cell densities $(0.5$ $\times 10^{6} \mathrm{vs} 1.0 \times 10^{6}$ ). In total, 180 explants of six horses (30 per horse) were divided into five groups: no lesion (i), lesion alone (ii), lesion with naïve MSCs (iii), lesion with chondrogenically-induced MSCs (iv) and lesion with chondrogenically-induced and PEMF-stimulated MSCs (v). Half of the explants were mechanically loaded and compared with the unloaded equivalents. Within each circumstance, six explants were histologically evaluated at different time points (day 1 ,
\end{abstract}

J.H. Spaas and S.Y. Broeckx share first authorship. A. Franco-Obregón and K. Wuertz-Kozak share last authorship.

Jan H. Spaas

and Karin Wuertz-Kozak

KARGER 125
Global Stem cell Technology, ANACURA group, Noorwegenstraat 4, 9940 Evergem, (Belgium), or Institute for Biomechanics, ETH Zurich, and Competence Center for Applied Biotechnology and Molecular Medicine CABMM, University of Zurich, Zurich, (Switzerland),E-Mail jan.spaas@anacura.com, E-Mail kwuertz@ethz.ch 
5 and 14). Results: COMP expression was selectively increased by chondrogenic induction ( $p=0.0488$ ). PEMF stimulation (1mT for 10 minutes) further augmented COL II expression over induced values $(p=0.0405)$. On the other hand, MSC markers remained constant over time after induction, indicating a largely predifferentiated state. In the unloaded group, MSCs adhered to the surface in $92.6 \%$ of the explants and penetrated into $40.7 \%$ of the lesions. On the other hand, physiological loading significantly reduced surface adherence (1.9\%) and lesion filling (3.7\%) in all the different conditions ( $p<0.0001)$. Remarkably, homogenous cell distribution was characteristic for chondrogenic induced MSCs (+/- PEMFs), whereas clump formation occurred in 39\% of uninduced MSC treated cartilage explants. Finally, unloaded explants seeded with a moderately low density of MSCs exhibited greater lesion filling ( $p=$ $0.0022)$ and surface adherence $(p=0.0161)$ than explants seeded with higher densities of MSCs. In all cases, the overall amount of lesion filling decreased from day 5 to $14(p=0.0156)$. Conclusion: The present study demonstrates that primed chondrogenic induction of MSCs at a lower cell density without loading results in significantly enhanced and homogenous MSC adhesion and incorporation into equine cartilage.

Copyright $\odot 2015$ S. Karger AG, Basel

\section{Introduction}

Osteoarthritis, also known as degenerative joint disease (DJD), is a pathology characterized by degenerative and sometimes hypertrophic changes of bone and cartilage, resulting in progressive apposing of joint surfaces causing pain and joint distortion. In the US between the years of 2010 and 2012, 52.5 million adults (22.7\%) demonstrated doctordiagnosed arthritis [1]. By 82 years of age, as high as 50\% of the population will develop knee osteoarthritis [2], with greater morbidity in modern societies [3]. Also in horses, 60\% of locomotory disorders are correlated with osteoarthritis [4], which is considered a major economic loss for the industry $[5,6]$.

The evolutionary distance between human and rhesus monkey genome is estimated to be around 35 million years, whereas this would be 80 million years for rodents [7]. Large animals, such as horses and dogs, are located somewhere in between [8]. Histological evaluation of horse, goat, sheep, dog, and rabbit stifle joint cartilage demonstrated closest proximity of equine stifle cartilage to its human equivalent [9]. Therefore, horses may be considered as a relevant pre-clinical animal model for human therapies. Also the FDA reported in 2005 the use of equine cartilage studies for assessing clinical endpoints in human clinical trials [10].

Recently, it has been shown that pulsed electromagnetic field (PEMF) treatment of injured rabbit knees can significantly improve histological scores and hyaline cartilage formation after 6 weeks of daily PEMF treatment for one hour a day [11]. In 28 elderly humans with bilateral knee osteoarthritis, PEMF stimulation at 3 times per week over 6 weeks significantly improved pain, stiffness, and physical function in comparison to the untreated contralateral joint [12]. Trock et al. found that treating human patients with knee osteoarthritis $(n=86)$ with PEMFs for a total of 18 times caused significant differences in pain, pain on motion, and tenderness in comparison to placebo treatments [13]. Interestingly, PEMF stimulation of human umbilical cord-derived mesenchymal stem cells (MSCs) was able to enhance proliferation and accelerate chondrogenic differentiation and extracellular matrix production in vitro [14] and similar beneficial effects were observed for adiposederived MSCs in both, 2D and 3D culture [15].

It was shown that experimentally induced cartilage lesions in horses treated with autologous adipose tissue or bone marrow-derived MSCs in combination with hyaluronic acid or fibrin hydrogels revealed only modest clinical improvements in comparison to the placebo treatment group, however, with enhanced tissue repair and increased ACAN levels [16-18]. Rather than using uninduced MSCs, several researchers have chosen to perform 


\section{Cellular Physiology Cell Physiol Biochem 2015;37:651-665 and Biol: DOI: 10.1159/000430384 2015 S. Karger AG, Basel and Biochemistry Published online: September 08, $2015 \quad$\begin{tabular}{l} 
www.karger.com/cpb \\
\hline
\end{tabular} \\ Spaas et al.: Chondrogenic Priming Enhances MSC Adhesion}

chondrogenic predifferentiation of MSCs, which resulted in enhanced therapeutic effects [19-21].

In order to provide more insights in the aforementioned findings, the present study consists of a series of combined cell culture and organ culture experiments. Firstly, equine peripheral blood (PB) MSCs were exposed to chondrogenic induction with and without PEMF treatment and changes in the mRNA expression of chondrogenic markers relative to untreated PB-MSCs was analysed after 6, 24 and 72 hours (n=3). In the second part, lesions were introduced into stifle joint cartilage explants of six horses, followed by topical application of allogeneic PB-MSCs obtained from one donor horse, at two different seeding densities. Five different groups were considered at three time points (day 1, 5 and 14) with or without loading: no lesion (i), lesion alone (ii), lesion with naïve MSCs (iii), lesion with chondrogenically-induced MSCs (iv) and lesion with chondrogenically-induced and PEMFstimulated MSCs (v).

\section{Materials and Methods}

\section{Cell Culture Study}

Isolation and expansion of MSCs. In total, $50 \mathrm{ml}$ of blood was collected in sterile EDTA tubes from the vena jugularis of three different adult donor horses, which were tested for different transmittable diseases at Böse laboratory (Harsum, Germany), as previously reported [22]. Approval of Global Stem cell Technology's ethics committee was obtained (EC_2012_001). In order to isolate mesenchymal stem cells (MSCs), the blood sample was centrifuged at $1000 \mathrm{G}$ for 20 minutes and the buffy coat was collected and diluted 1:2 in phosphate buffered saline (PBS) 1x. Afterwards, this suspension was gently layered on an equal amount of Percoll ${ }^{\circledR}$ density gradient (GE Healthcare) and isolated cells were further cultured in expansion medium consisting of DMEM low glucose $+20 \%$ FCS +50 units $/ \mathrm{ml}$ penicillin, $50 \mu \mathrm{g} / \mathrm{ml}$ streptomycin and $125 \mathrm{ng} / \mathrm{ml}$ amphotericin at $37^{\circ} \mathrm{C}$ and $5 \% \mathrm{CO} 2$ and characterized as MSCs [23]. During the expansion phase, the culture medium was changed every two days and cells were subcultured at $70 \%$ confluency with $0.25 \%$ trypsin. All three horses were used in the cell culture study, whereas a single donor horse with the highest MSC yields was used as a source of allogeneic stem cells for the organ culture study.

For quality control purposes, an immunophenotypic characterization [23] of all donor MSCs was performed at passage 5 (P5) and P10. Briefly, untreated as well as chondrogenically-induced MSCs were tested by flow cytometry for the following stem cell markers: mouse anti-human CD29-APC (Biolegend), mouse anti-horse CD44-FITC (AbD Serotec) and mouse anti-horse CD90 PE-Cy7 (VMRD); and for the adult blood cell (negative) markers: mouse anti-human CD45 RPE-Cy5.5, mouse anti-horse major histocompatibility complex (MHC) type II-PE and monocyte/macrophage mouse anti-human macrophagesAlexa 488 (all from AbD Serotec). Cross reactivity of negative markers with equine epitopes was assessed using equine peripheral blood mononuclear cells as previously reported [23].

Stimulation of MSCs (chondrogenic induction, PEMFs). At P4, MSCs were seeded for either standard expansion, chondrogenic induction or chondrogenic induction in combination with stimulation with pulsed electromagnetic fields (PEMFs). Cells were chondrogenically induced as previously reported $[19,20]$ by culturing $6.7 \times 10^{3} \mathrm{MSCs} / \mathrm{cm}^{2}$ until the next confluency in a medium consisting of DMEM LG, 20\% FCS, 1\% AB/AM (Penicillin-Streptomycin-Amphotericin B), $1.7 \times 10^{-4} \%$ insulin-like growth factor (IGF)-I and 1.0x10${ }^{5} \%$ transforming growth factor (TGF)- $\beta$. The seeding protocol was dependent on the type of study (see below):

For the cell culture study (Fig. 1) that aimed at detecting the effect of chondrogenic induction and PEMF treatment on the expression of cartilage-specific markers, MSCs were seeded at a density of $1 \times 10^{4}$ MSCs $/ \mathrm{cm}^{2}$ in T25 flasks. For the organ culture experiments in which the aim was to analyse the regenerative capacity of MSCs as a function of treatment (induction, PEMF, loading, seeding density), MSCs were seeded at a density of $1 \times 10^{4} \mathrm{MSCs} / \mathrm{cm}^{2}$ in T75 flasks. In order to obtain a sufficient number of cells for seeding 2 different cell densities onto created lesions $\left(0.5\right.$ or $1 \times 10^{6}$ MSCs per cartilage sample, 9 cartilage samples to be treated with MSCs), nine T75 flasks were prepared for each of the 3 conditions. The experimental set-up is illustrated in Fig. 1. 
Fig. 1. Study outline assessing peripheral blood-derived MSCs in a cell and organ culture study.

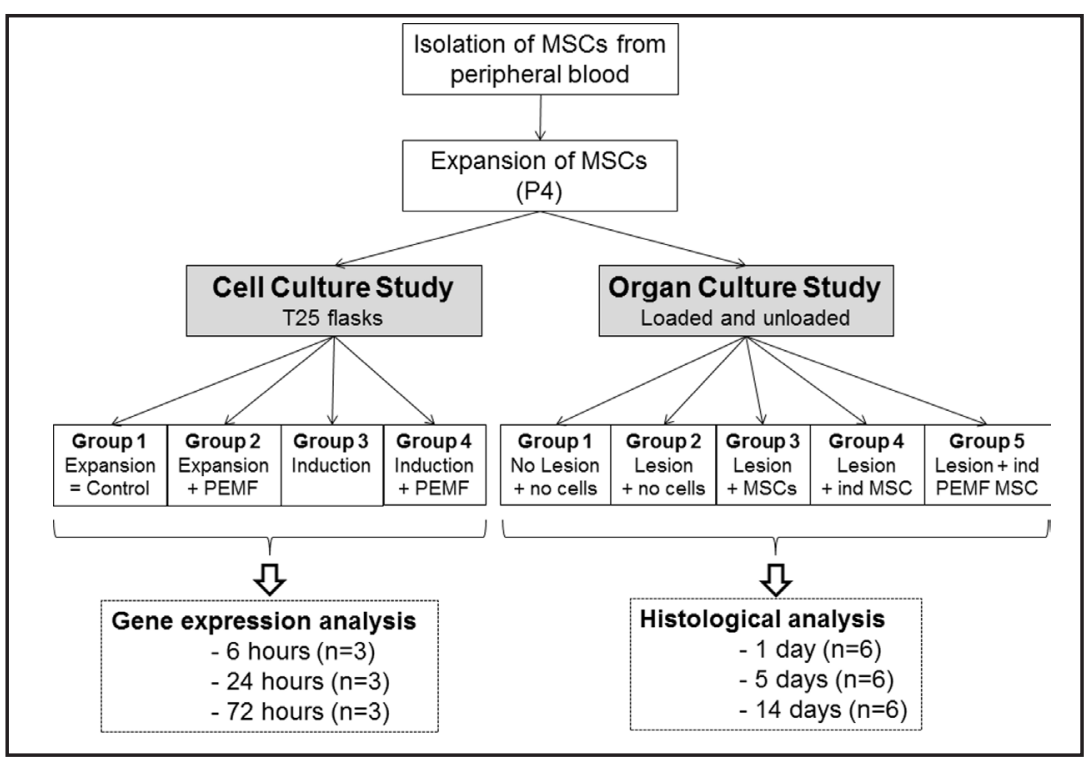

For both experiments (cell/organ culture), PEMF stimulation at $1 \mathrm{mT}$ and $0.5 \mathrm{mT}$ was applied to the respective samples for a single exposure of 10 minutes using a custom-made device [24]. Growth factorbased chondrogenic induction was conducted as previously described $[19,20]$.

Analysis of changes in chondrogenic marker gene expression. In the cell culture study, changes in the gene expression of the chondrogenic markers oligomeric matrix protein (COMP), aggrecan (ACAN) and collagen type II (COL II) upon stimulation of MSCs with chondrogenic medium with or without PEMF were analysed by qPCR. As indicated in Fig. 1, MSCs were cultured in normal expansion medium or induction medium for 6, 24 or 72 hours of culture before being lysed in $1 \mathrm{ml}$ of Trizol (Invitrogen) and RNA was isolated by standard chloroform phase separation ( $250 \mu \mathrm{l}$, Sigma-Aldrich) as previously described [25]. RNA was quantified on the Nanodrop Lite (Fisher Scientific) before reverse transcribing $1 \mu \mathrm{g}$ of RNA into cDNA ( 10 minutes at $25^{\circ} \mathrm{C}, 120$ minutes at $37^{\circ} \mathrm{C}$ ), using the TaqMan Reverse Transcription Reagents Kit (Life Technologies). cDNA was mixed with equine specific primers (Applied Biosystems, Assays on Demand: COMP = Ec03468079_g1, ACAN = Ec03469667_m1, COL II = Ec03467386_g1, GAPDH = Ec03210916_gH) and PCR Fast Master Mix (Applied Biosytems) and gene expression was analysed in triplicate on the Biorad CFX96 Touch Real-Time PCR detection system as previously reported [19].

Values were normalized to GAPDH (house-keeping gene) and are presented as fold change relative to uninduced MSCs (i.e. MSCs in expansion medium without PEMF treatment), using the comparative CT method $\left(=2^{-\Delta \Delta C T}\right.$ method $)$.

\section{Organ Culture Study}

PKH 26 labeling of MSCs. For the organ study, MSCs were cultured in normal expansion medium or induction medium in the dedicated samples as indicated in Fig. 1. Upon $60 \%$ confluency, MSCs were harvested by trypsin treatment and fluorescently labeled with the PKH26 Red Fluorescent Cell Linker Kit for General Cell Membrane Labeling according to the manufacturer's protocol (Sigma-Aldrich) to allow long-term cell tracking upon seeding onto cartilage samples. Briefly, MSCs from one treatment condition were pooled, centrifuged at $300 \mathrm{G}$ for 8 minutes, counted by hemacytometer and aliquoted into nine cell suspension portions ( $=3$ horses per experiment at 3 time points per treatment) of either 0.5 or $1 \times 10^{6} \mathrm{MSCs}$ each (to be seeded onto one cartilage explant each). After centrifugation, individual MSC samples were resuspended in $50 \mu \mathrm{l}$ of DILUENT C, followed by addition of the same volume of PKH26 stain and incubated for 5 minutes with periodic mixing. After incubation, $100 \mu \mathrm{l}$ of FCS was added to block the reaction and labelled MSCs were retrieved by centrifugation ( 400 G, 5 minutes) following one wash cycle with PBS.

In a pilot study, the sustainability of the PKH26 labelling of the herein used cell type was examined and confirmed in 2D culture for up to 18 days (data not shown).

Sampling and incision of cartilage explants. At the slaughter house cartilage explants were aseptically excised from the patellar cartilage from six slaughtered horses between 4 and 21 years-old (4 mares and 2 


\section{Cellular Physiology Cell Physiol Biochem 2015;37:651-665

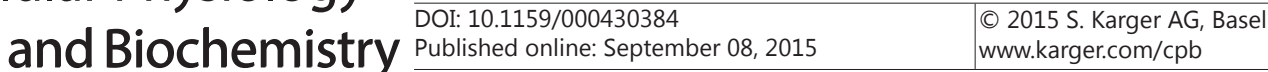 \\ Spaas et al.: Chondrogenic Priming Enhances MSC Adhesion}

geldings) using an $8 \mathrm{~mm}$ biopsy punch ( $\mathrm{n}=30$ per horse). The biopsies were removed from the subchondral bone with a scalpel blade, placed separately in $15 \mathrm{ml}$ tubes containing sterile PBS plus penicillin (100 units/ $\mathrm{ml})$, streptomycin $(100 \mu \mathrm{g} / \mathrm{ml})$ and amphotericin $(250 \mathrm{ng} / \mathrm{ml})$ and transported to the laboratory. Samples were rinsed with the same solution under the laminar flow and individually placed in 24 well plates with $1 \mathrm{ml}$ of expansion medium (see above) to keep samples hydrated. One explant at a time from the lesion groups was removed from the well, positioned on a sterile gauze and treated with a $1.5 \mathrm{~mm}$ deep incision in the centre of the biopsy ( 2 parallel cuts of $4 \mathrm{~mm}$ length each, plus 2 identical cuts in perpendicular plane as shown in Fig. 2), created through a number 15 scalpel blade with a custom-made holder to control depth of incision. On top of the front side of each biopsy, an oblique $3 \mathrm{~mm}$ punch biopsy lesion was created for histological orientation.

MSC application onto cartilage explants. Cells were washed once, centrifuged at $400 \mathrm{G}$ for 5 minutes and resuspended at a dose of 0.5 or $1 \times 10^{5} / \mu \mathrm{l}$ in $10 \mu \mathrm{l}$ of bioreactor medium (expansion medium with penicillin (100 units $/ \mathrm{ml})$, streptomycin $(100 \mu \mathrm{g} / \mathrm{ml})$ and amphotericin $(250 \mathrm{ng} / \mathrm{ml}))$.

Biopsies were positioned into 24-well plates containing $200 \mu \mathrm{l}$ of bioreactor medium, with the injured side (if applicable) facing upwards. $1 \times 10^{6}$ of the PKH 26 labelled MSCs from the respective treatment groups were resuspended in $10 \mu \mathrm{l}$ of expansion medium and slowly seeded on top of their respective explant. The other half of the explants were covered with $0.5 \times 10^{6}$ cells in $10 \mu$ of expansion medium. In cell-free samples, $10 \mu \mathrm{l}$ of pure expansion medium was added to the explant. After allowing MSCs to attach to the explants for approximately $20 \mathrm{~min}$ in the incubator, $800 \mu \mathrm{l}$ of bioreactor medium was carefully added per well in order to cover the whole sample with medium.

Fourteen hours later, the explants were brought to new wells with fresh media and non-adhered cells were visualized and photographed using a fluorescence microscope. In order to determine the percentage of MSCs that attached to the cartilage explant, the media of the old wells was collected and cells attaching to the plastic surface were harvested by trypsin treatment and pooled with the culture medium, followed by cell counting.

All cartilage explants were positioned in new wells with fresh medium each day and maintained at $37^{\circ} \mathrm{C}$ with $5 \% \mathrm{CO}_{2}$, independent of whether mechanical loading was applied, or not (see below). The remaining 24-well plates were analysed each day for floating and detached cells.

Loading of cartilage explants. 40 hours after the initial seeding, half of the cartilage explants that were assigned to daily physiological loading were exposed to their first loading cycle at $37^{\circ} \mathrm{C}$, while non-loaded samples were maintained in the incubator. For physiological dynamic loading of cartilage explants, a custommade bioreactor was used that allows simultaneous loading of six specimens at a time [26]. Briefly, cartilage explants were positioned between two metal plates of a sterile loading device positioned in 50 ml Falcon tubes containing $20 \mathrm{ml}$ of bioreactor medium to fully cover the specimens throughout the loading phase. Dynamic loading was performed on the Instron E10000 (Instron International) for 1 hour a day at $40 \mathrm{~N}$ per explant ( $1 \mathrm{~Hz}$ ) and the same time point each day. After 1, 5 and 14 days, the respective samples (as well as the unloaded samples) were fixed for histological analysis (see below).

Histological scoring of cartilage explants. At the respective time point (day 1, 5 and 14), the specimens were fixed in neutral buffered $10 \%$ formalin, embedded in paraffin, sectioned at $4 \mu \mathrm{m}$ thickness and stained

Fig. 2. $8 \mathrm{~mm}$ cartilage punch biopsies of $3 \mathrm{~mm}$ thickness were removed from the equine femoropatellar joint (A). Two parallel cuts of $4 \mathrm{~mm}$ length were created in the horizontal and vertical plane creating a central grid (B). On top of the front side of each biopsy, an oblique $3 \mathrm{~mm}$ punch biopsy lesion was created for histological orientation (B).

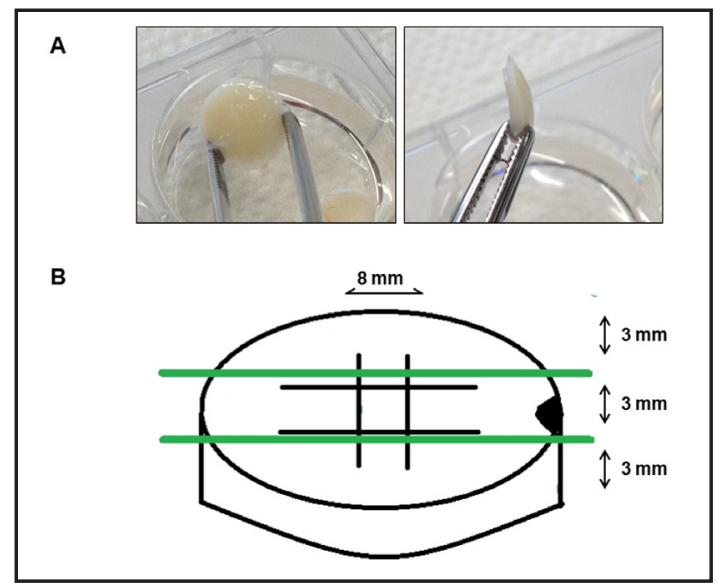




\section{Cellular Physiology Cell Physiol Biochem 2015;37:651-665 \\ \begin{tabular}{l|l|l} 
DOI: 10.1159/000430384 & (C) 2015 S. Karger AG, Basel
\end{tabular} \\ and Biochemistry Published online: September 08, $2015 \quad$ www.karger.com/cpb \\ Spaas et al.: Chondrogenic Priming Enhances MSC Adhesion}

with Hematoxylin \& Eosin, Safranin 0 and Alcian Blue. All sections were blindly scored by an anatomical pathologist $(\mathrm{RF})$. The following parameters were considered: lesion presence $(0=$ not present or $1=$ present); surface cell adhesion $(0=$ not present or $1=$ present $)$; cell integration $(0=$ not present or $1=$ present); number of cell layers; chondrocyte necrosis ( $0=$ no, $1=$ moderate or 2 = severe); chondrone formation grade $(0=$ no, $1=$ moderate or $2=$ excessive); chondrone density $(1=2$ cell layers, $2=2$ to 5 cell layers, $3=$ more than 5 cell layers $)$; lesion filling $(0=$ no, $1=$ moderate or $2=$ extensive $)$; clump presence $(0$ $=$ not present or 1 = present) and number of clump layers.

\section{Statistical analysis}

For the cell culture study, gene expression of treatment groups (induction, PEMFs) was analysed based on a mixed model with horse as random effect and time, medium and/or PEMF stimulation and their two-way interaction as categorical fixed effects. The F-test at 5\% significance level and Tukey's adjustment method were used for multiple comparisons.

For the organ culture study, the stratified Wilcoxon rank sum test for response variables with more than two levels and the stratified McNemar test for response variables only two levels were used. Loaded explants were compared with unloaded explants stratifying for horse, treatment and time. Treatments were compared stratifying for horse and time and only using data without loading. Finally, time points were compared stratifying for horse and treatment and only using data without loading.

In order to compare the two doses, Student T-test was used to compare the average horse values (averaged over time, treatment and loading).

In all analyses, a significance level of $5 \%$ was used.

\section{Results}

Cell Culture Study

MSC response to chondrogenic induction. The effects of chondrogenic induction medium were analysed on the gene expression level (mRNA expression of COMP, ACAN and COL II) as well as on the protein level (Alcian Blue staining, counterstained with Hematoxylin for visualizing unstained cells). PCR revealed an increase in cartilage specific genes after chondrogenic induction of the MSCs in three different horses (Fig. 3A). While ACAN expression increased only 2-3 fold upon exposure to induction medium, which was not statistically significant $(p=0.2300)$, COMP expression significantly increased $\sim 5-6$ fold ( $p$ $=0.0488$ ). In contrast, no considerable effects were observed for COL II at any of these early time points, indicating limited chondrogenic induction (Fig. 3A).

Flow cytometry revealed no significant changes in the percentage of positive (CD29, CD44 and CD90) and negative (CD45, MHC II and monocyte/macrophage marker) stem cell markers over time $(\mathrm{p}=0.37)$ nor after chondrogenic induction at P5 $(\mathrm{p}=0.51)$ and P10 (p $=0.28$ ) in all horses (Fig. 4A \& B). This confirmed maintenance of MSC immunophenotypic properties over the course of 72 hours, hence supporting the notion of early chondrogenic induction. A spindle-shaped morphology could be noticed in uninduced MSC cultures (Fig. 4C), whereas chondrogenic induced MSCs displayed a more rectangular morphology (Fig. 4D). Alcian Blue staining confirmed the presence of glycosaminoglycans in the chondrogenicinduced group (Fig. 4D), whereas no blue staining was observed in MSCs cultured in expansion medium (Fig. 4C).

MSC response to PEMF. COL II expression in MSC cultures exposed to $1 \mathrm{mT}$ of PEMFs for 10 minutes increased significantly $(\mathrm{p}=0.0405)$ after 24 hours in chondrogenic induction medium (Fig. 3B). PEMF exposure of MSCs cultured in expansion medium at either 0.5 or $1 \mathrm{mT}$ amplitudes did not result in a significant increase $(\mathrm{p}=0.8521)$ in any of the evaluated cartilage genes (i.e. ACAN, COL II and COMP) at any of the time points in comparison to unstimulated MSCs (Fig. 3C presents 24 hour time point as example). In contrast with $0.5 \mathrm{mT}$, the $1 \mathrm{mT}$ stimulated MSCs cultured in chondrogenic induction medium resulted in a significant increase in COL II expression (Fig. 3D), revealing a culture medium- and PEMF dose-dependence. Hence, at 24 hours PEMF stimulation was able to significantly enhance 


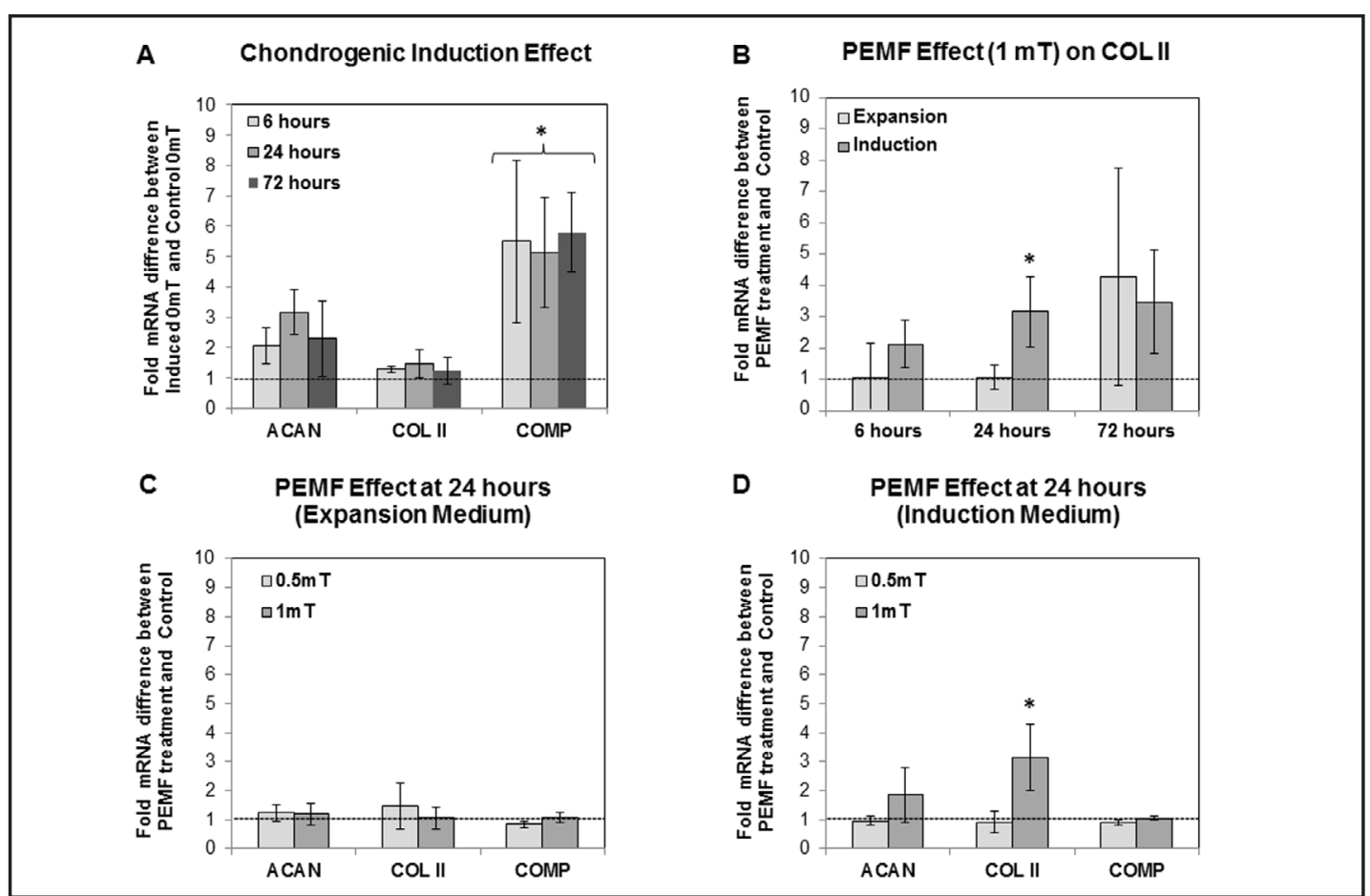

Fig. 3. RT-PCR for gene expression of ACAN, COL II and COMP. Increase of cartilage gene expression after chondrogenic induction in comparison to uninduced MSCs (A). Effect of PEMF on COL II expression of uninduced and chondrogenic induced MSCs at different time points (B). Effect of $0.5 \mathrm{mT}$ and $1 \mathrm{mT}$ of PEMF stimulation on the expression of different cartilage genes in uninduced (C) and chondrogenic induced MSCs (D) at 24 hours. Values are given as the mean of three measurements \pm SEM; * indicates $\mathrm{p}<0.05$.

COL II expression. We next tested the ability of the combination of PEMFs and induction to improve the regenerative capacity of MSCs in an organ culture.

\section{Organ Culture Study}

PKH26 labelling and cell adhesion. PKH26 labelled MSCs could be visualized in more than $90 \%$ of the cells until day 18 after culturing in control wells, indicating a suitable staining for the 14 day explant experiment. At 14 hours after adding the cells on the explants, non-adhered cells clearly contained the fluorescent label as confirmed by fluorescence microscopy analysis (Fig. 5). Table 1 indicates the percentage of cells that initially did not attach to the cartilage explants of the six horses before loading, shown separately for each study group. In explant cultures designated for loading, an average of $38.6 \pm 14.0 \%$ of the cells was not able to attach (Table 1). For unloaded explants this remained within the same range $(35.9 \pm 12.5 \%)$. In the low $\left(0.5 \times 10^{6} \mathrm{MSCs}\right)$ and high $\left(1 \times 10^{6} \mathrm{MSCs}\right)$ seeding density groups, an average of $41.3 \pm 12.6 \%$ versus $33.1 \pm 12.8 \%$ of the cells did not attach to the explants, respectively (Table 1). No significant differences in cell losses before loading were found between explants treated with high and low density of MSCs. After 24 hours, no more cells were detected in the supernatant or at the bottom of the wells. At each time point, adhered MSCs were visualized with a fluorescence microscope; however, no more red fluorescent signal could be detected after tissue sectioning.

Histological results of loaded cartilage explants. Daily compressive physiological loading was applied to cartilage explants in order to enhance the in vivo comparability. Although loading was only as high as $40 \mathrm{~N}$ for 1 hour per day at $1 \mathrm{~Hz}$, it had severe effects on the presence of MSCs (independent of the pre-treatment of cells). Taking all the time points together, after exposure to loading, only $1.9 \%$ or 1 out of 54 (= 3 time points $\times 6$ horses $\mathrm{x}$ 3 MSC treatment groups) cartilage explants demonstrated adherent MSCs on their surface 


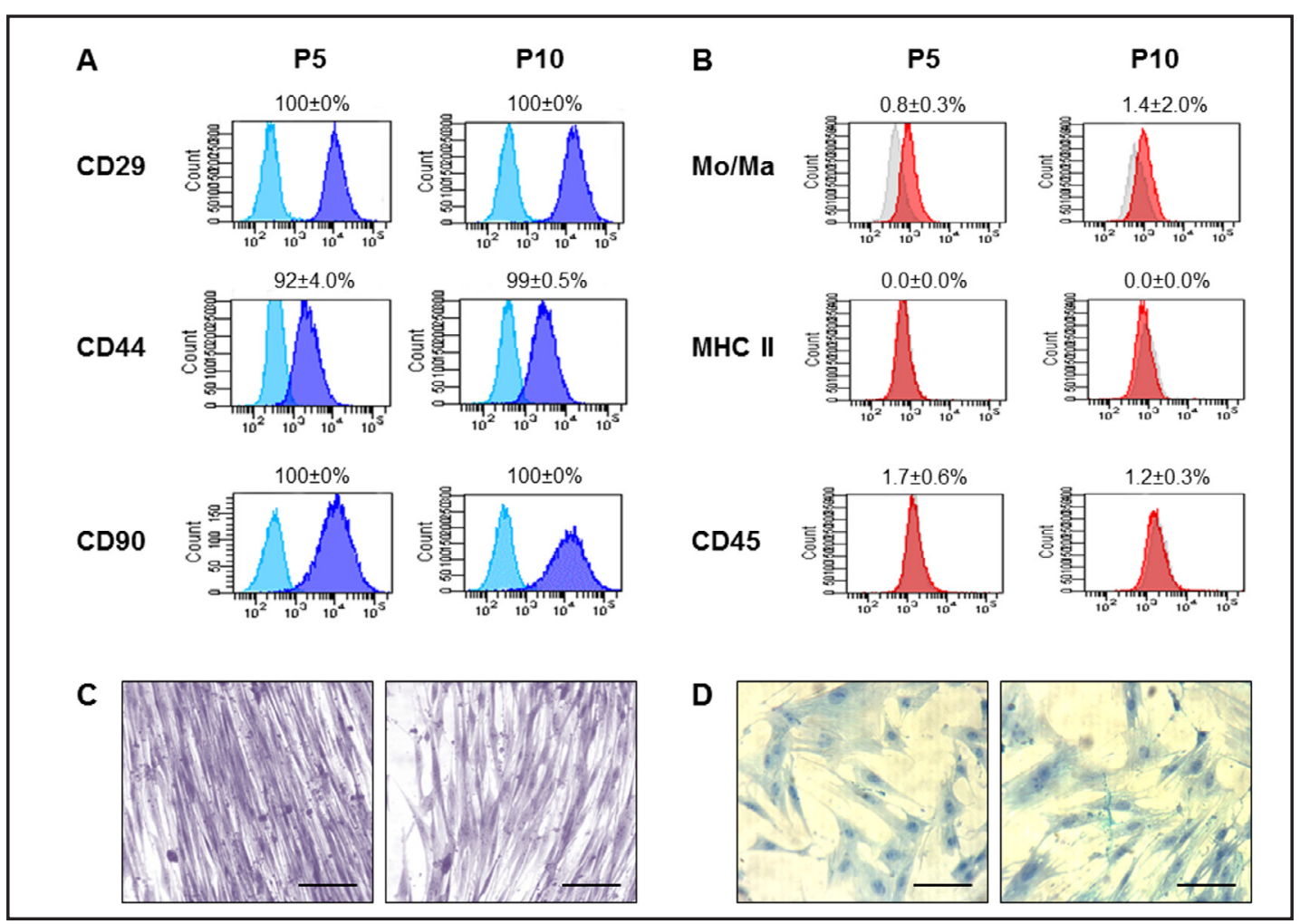

Fig. 4. Flow cytometry confirmed a positive expression for CD29, CD44 and CD90 compared to isotype control (A), whereas no expression for a monocyte/macrophage marker, major histocompatibility complex (MHC) type II and CD45 could be observed at P5 and P10 on chondrogenic induced MSCs (B). Light microscopic images of MSCs in their undifferentiated state (C) and chondrogenic induced state (D) after Alcian Blue staining. Glycosaminoglycan production can be noticed after induction. Scale bars represent $50 \mu \mathrm{m}$.

Fig. 5. Phase contrast (A), fluorescence (B) and overlay (C) images of PKH26-labelled MSCs in the different treatment groups at 14 hours after seeding: uninduced (I), chondrogenic induced (II) and PEMF stimulated chondrogenic induced (III) MSCs. Scale bars represent $50 \mu \mathrm{m}$.

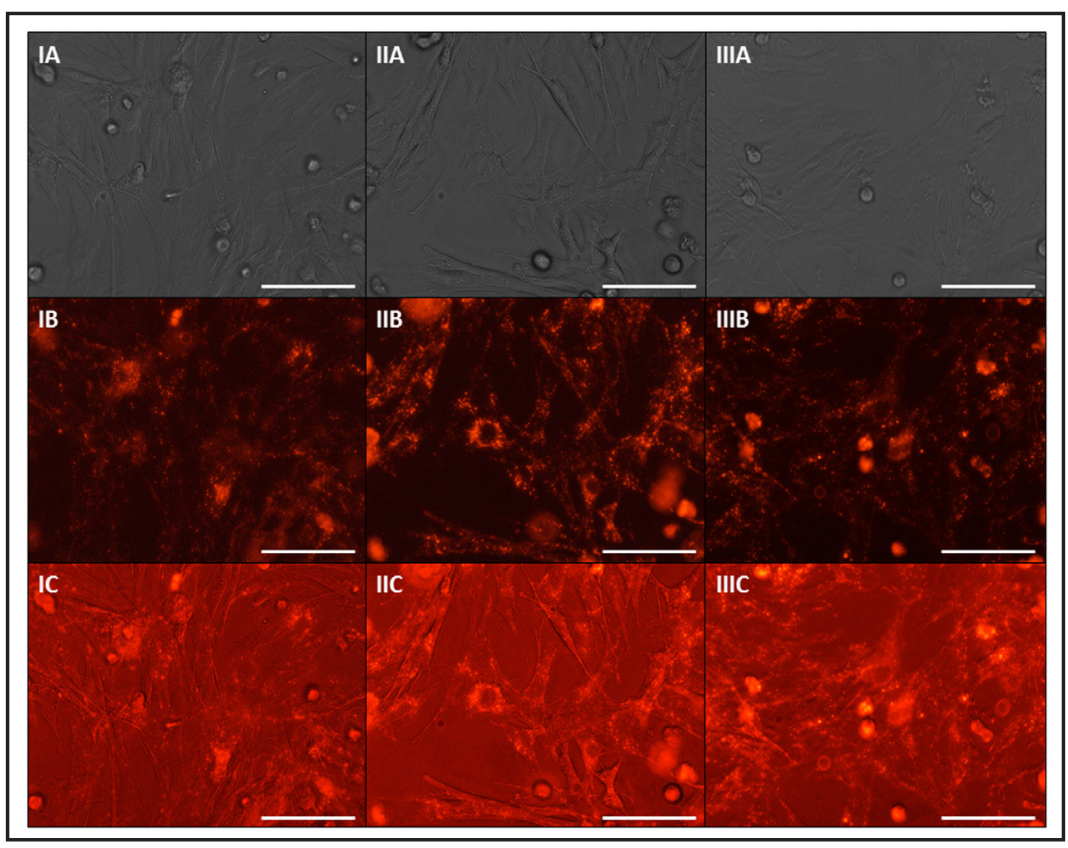

(Fig. 6) which resulted in absence of clump formation in the loaded group. Consequently, lesion filling was only observed in $3.7 \%$ or 2 out of 54 cartilage explants (Fig. 6).

\section{KARGER}


Table 1. Percentage of cells that initially did not attach on the loaded (L) and unloaded (U) cartilage explants at 14 hours after seeding and before the first loading session

\begin{tabular}{|c|c|c|c|c|c|c|c|c|c|c|c|c|}
\hline & \multicolumn{6}{|c|}{$1 \times 10^{6}$ CELLS PER EXPLANT } & \multicolumn{6}{|c|}{$0.5 \times 10^{6}$ CELLS PER EXPLANT } \\
\hline & \multicolumn{2}{|c|}{ Horse 1} & \multicolumn{2}{|c|}{ Horse 2} & \multicolumn{2}{|c|}{ Horse 3} & \multicolumn{2}{|c|}{ Horse 4} & \multicolumn{2}{|c|}{ Horse 5} & \multicolumn{2}{|c|}{ Horse 6} \\
\hline & $\mathrm{L}$ & $\mathrm{U}$ & $\mathrm{L}$ & $\mathrm{U}$ & $\mathrm{L}$ & $\mathrm{U}$ & $I$ & $\mathrm{U}$ & $\mathrm{L}$ & $\mathrm{U}$ & $\mathrm{L}$ & $\mathrm{U}$ \\
\hline & 18 & 23 & 54 & 50 & 50 & 47 & 30 & 18 & 54 & 54 & 54 & 48 \\
\hline Chondro Induced & 23 & 23 & 18 & 32 & 50 & 45 & 30 & 18 & 54 & 54 & 42 & 30 \\
\hline Induced + PEMFs & 32 & 18 & 18 & 41 & 32 & 27 & 30 & 36 & 54 & 36 & 54 & 48 \\
\hline
\end{tabular}

Fig. 6. Representative images of loaded versus unloaded cartilage explants at 40 -fold magnification. Different groups are illustrated: no lesion without MSCs (A), only a lesion without MSCs (B), lesion with MSCs (C), lesion with chondrogenic induced MSCs (D) and lesion with PEMF stimulated chondrogenic induced MSCs (E). Boxes represent 200 fold magnifications.

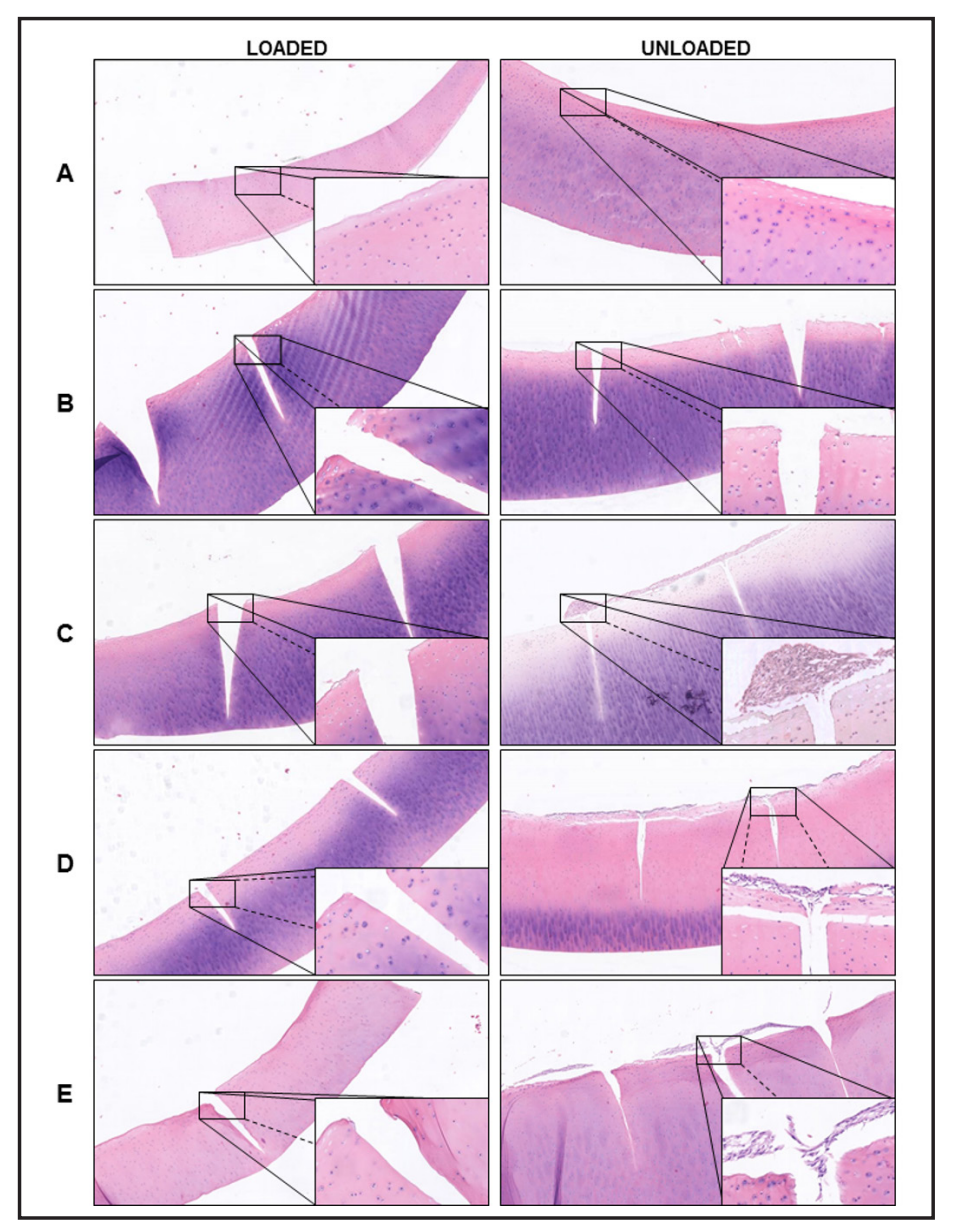

Histological results of unloaded explants. Taking all three time points of unloaded MSCtreated groups (iii, iv and v) together, adherent MSCs were present in $92.6 \%$ of the explants $(=50 / 54=($ Day $1=18 / 18)+($ Day $5=17 / 18)+($ Day $14=15 / 18))$, which is significantly more than $1.9 \%$ in the loaded explants $(\mathrm{p}<0.0001)$. It made it also possible to histologically score and compare the different unloaded treatment groups. For all the scored parameters, no significant differences between chondrogenically-induced MSCs with or without PEMF stimulation could be demonstrated. On the other hand, in chondrogenic induced MSC groups (with or without PEMF), a homogenous cell distribution and no cell clumps were more characteristic, whereas 7 out of 18 (39\%) uninduced MSC treated cartilage explants exhibited cell clumps of at least 8 cell layers thickness. Clumping incidence was significantly different from the other treatment groups $(\mathrm{p}=0.0156)$ and was independent of cell seeding number. The number of explants with lesion filling $(40.7 \%=22 / 54)$ significantly decreased $(\mathrm{p}=0.0156)$ in the unloaded group from day $5(\mathrm{n}=11 / 18)$ to day $14(\mathrm{n}=4 / 18)$, in contrast to what was observed from day $1(\mathrm{n}=7 / 18)$ to $5(\mathrm{p}=0.2188)$. Although no more clumps were noticed in any explant culture at day 14 (Fig. 7), the number of explants that demonstrated adhered MSCs on their surface did not significantly alter over time $\left(\mathrm{p}_{1 \rightarrow 5}=1.000, \mathrm{p}_{5 \rightarrow 14}=\right.$ 
Fig. 7. Representative images of unloaded explants at a 40 and 200 fold magnification. Cartilage explants treated with uninduced MSCs contain clumps at day 1 (A) and day 5 (B), whereas clumps detached before day 14 (C). Cartilage explants after treatment with chondrogenic induced MSCs demonstrate a homogenous cell adhesion which remains constant from day 1 (D), towards day 5 (E) and day 14 (F).

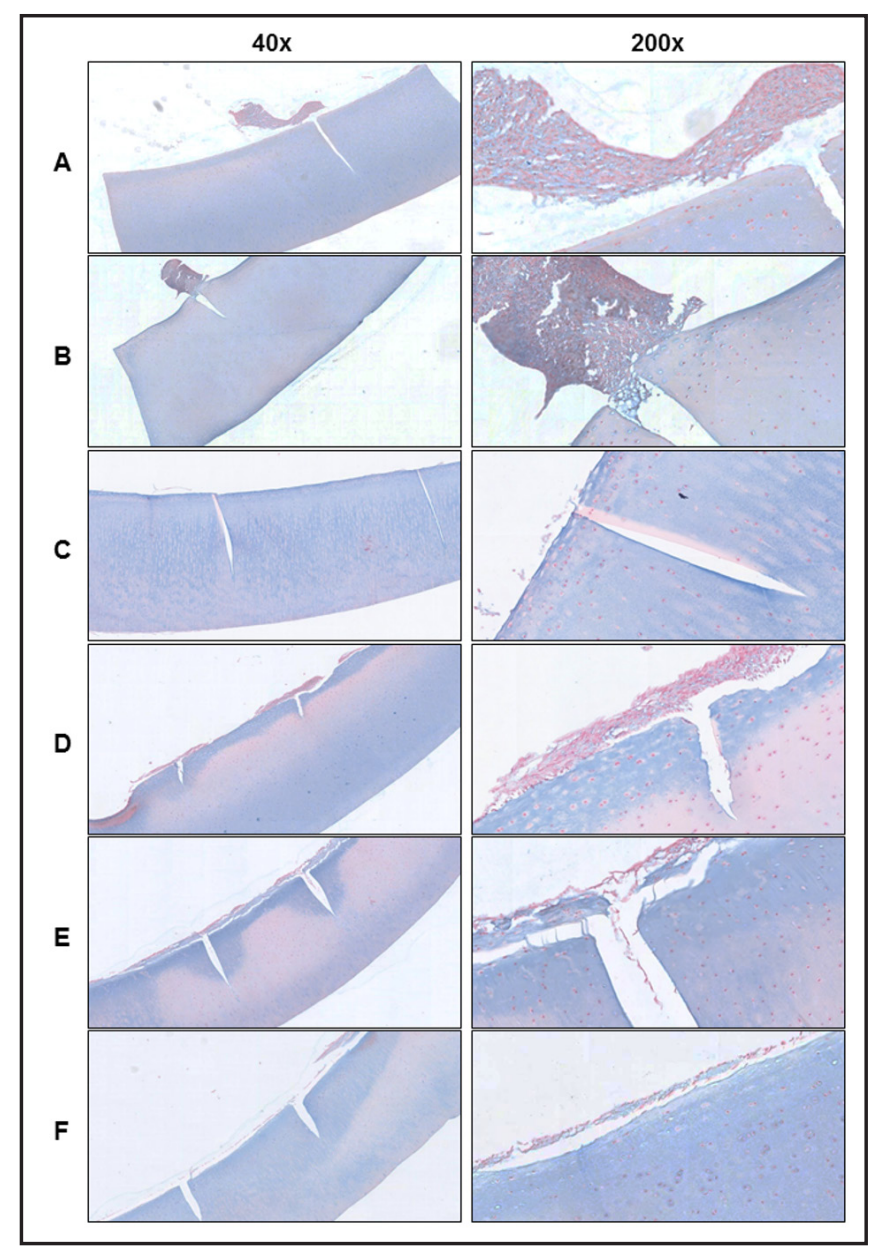

0.6250 , Fig. 7). The adhered non-clumped cell layers also remained relative constant over time $\left(\mathrm{p}_{1->5}=1.000, \mathrm{p}_{5-14}=0.1250\right.$, Fig. 7).

Taking all treatment scores in unloaded conditions together for the three horses treated with $0.5 \times 10^{6}$ MSCs (low density) versus the other three horses treated with $1 \times 10^{6}$ MSCs (high density), significantly more cartilage explants demonstrated lesion filling ( $p=0.0022)$ and surface adhered MSCs ( $p=0.0161$ ) in the low density group. The average score for lesion filling was 0.67 per low density treated cartilage explant and 0.15 for the high density treated explants. Concerning adhered MSCs, an average score of 1.00 demonstrated presence of MSCs on the surface of each low density explant, whereas this was not the case for the high density MSC application (average score of 0.85 ). On the other hand, no significant difference concerning integration $(p=0.6779)$, cell layers $(p=0.6974)$, clump formation $(p=0.6433)$ and clump layers $(p=0.6340)$ could be noticed between both.

\section{Discussion}

In the present study, peripheral blood (PB)-derived mesenchymal stem cells (MSCs) of three horses were successfully isolated and chondrogenically induced as confirmed by PCR and histology. Briefly, flow cytometry confirmed no loss of the typical MSC markers during the predifferentiation process. Half of the 180 cartilage explants were loaded in order to assess the cartilage integrity and integration capacities of fluorescently labelled MSCs under physiological compression at three time points (day 1, 5 and 14). Proliferation and differentiation are largely mutually exclusive processes in chondrogenesis. Chondrogenic 


\section{Cellular Physiology Cell Physiol Biochem 2015;37:651-665 \begin{tabular}{l|l} 
and Biochemistry Published online: September 08, 2015 & $\begin{array}{l}\text { C 2015 S. Karger AG, Basel } \\
\text { www.karger.com/cpb }\end{array}$ \\
\hline
\end{tabular} \\ Spaas et al.: Chondrogenic Priming Enhances MSC Adhesion}

induction was performed for only three days in a 2-dimensional culture system in order to maintain MSC proliferative capacity in an injectable cell-based product undergoing the initial stages of differentiation. The conservation of MSC markers and limited cartilage gene upregulation both indicate that the cells were in a prechondroblastic stage and not fully differentiated yet.

In order to track MSCs, labelling with the membrane incorporating substance PKH26 was performed, which was considered a suitable alternative to intranuclear labelling methods (e.g. green fluorescent protein GFP labelling) due to its well-described low toxicity and labelling sustainability in progenitor-like cells [27-30]. A preliminary experiment on PB-MSCs in 2D culture confirmed detectability of the fluorescent signal until day 18 (data not shown), yet moderately diluted due to cell division, as previously reported for pig chondrocytes [31]. Although successful fluorescent labelling of MSCs with PKH26 could be confirmed at the beginning of the organ culture study (on day 1 after seeding), the fluorescent signal could not be detected in the histological sections of the seeded cartilage explants. In contrast, other studies have previously described successful fluorescence-based detection of implanted, PKH-labelled MSCs and chondrocytes after 6 weeks (sheep) [32] or 12 weeks (mice) [31] in vivo, respectively. The exact reasons for the loss of fluorescent signal will have to be investigated in detail in future studies, but may be related to differences in the experimental condition and histological processing.

Although fluorescence labelling of MSCs was not detectable in the cartilage explants, MSCs could be identified based on their typical spindle-shaped morphology as well as by immunohistochemistry staining for MSC markers (data not shown). The spindle-shaped morphology at each analysis time point (after adding to the explants) indicates that MSCs did not attain fully differentiated chondrogenic features during two weeks of explant culture. This might be explained by the brief culture period (before adding to the explants) and the fact that, after a three-day induction period, no more cartilage inducing growth factors were used. A previous study using cartilage specific medium reports chondrogenic differentiation of integrated porcine bone marrow-derived MSCs after 6 weeks of culture in cartilage explant defects coated with agarose hydrogels [33].

In this study, the effects of chondrogenic induction, PEMF treatment, seeding density and mechanical loading on MSC adherence and functionality were analysed. After mechanical loading, significantly ( $\mathrm{p}<0.0001$ ) less explants exhibited lesion filling (3.7\% vs $40.7 \%)$ or cell adherence (1.9\% vs $92.6 \%$ ). As physiological pressures (ca. $0.8 \mathrm{MPa}$ ) were applied that are also expected to arise during moderate training, this finding indicates that mechanical loading - if applied soon after implantation/injection - may mechanically interfere with the integration of MSCs into isolated explants and undermine a potentially beneficial outcome. Cartilage contact pressures of $0.8 \mathrm{MPa}$ can be considered to be in the range of that acting in vivo on e.g. the equine stifle joint during normal ambulation, as peak pressures up to 1.5 - 2.0 MPa have been measured by Fowlie et al. [34]. Due to the weight and physiology of equine joints together with the robustness of equine subchondral bone [35], our findings may expose some restrictions for future clinical application. However, it has been reported that joint loading is highly dependent on the joint type and joint compartment [36]. In the present study, patellar cartilage was taken and it has been reported that this compartment is relatively unloaded during stall confinement and supervised walking, which could explain the sensitivity to loading $[36,37]$. The weight-bearing medial and lateral compartments on the other hand experience more in vivo loading, which might compromise the treatment. Further in vitro bioreactor tests with different loading patterns as well as tests under physiological circumstances will need to be performed to eventually make exercise recommendations for MSC-treated OA patients.

Chondrogenically-induced MSCs displayed superior adhesion and integration capacity in comparison to uninduced MSCs in this study. Homogenous layers of chondrogenic induced MSCs could be noticed all over the explants, whereas in 39\% of the uninduced MSCs large clumps of at least 8 cell layers were observed predominantly at areas on top of the explants which detached before day 14. Overall, a more efficient cell adhesion and integration 


\section{Cellular Physiology Cell Physiol Biochem 2015;37:651-665 \\ \begin{tabular}{l|l} 
DOI: 10.1159/000430384 & (C) 2015 S. Karger AG, Basel
\end{tabular} \\ and Biochemistry Published online: September 08, $2015 \quad$ www.karger.com/cpb \\ Spaas et al.: Chondrogenic Priming Enhances MSC Adhesion}

could be noticed in explants seeded with chondrogenic induced MSCs. On the other hand, the adhered non-clump cell layers remained relative constant over time, independent of predifferentiation. The beneficial effect of predifferentiation has also been reported in vivo in 165 horses, where clinical outcomes were improved upon chondrogenic predifferentiation [20].

Seeding density also had a significant effect on the outcome (in unloaded specimens). Interestingly, low cell densities $(27$ replicates $=3$ horses, 3 treatment circumstances at 3 time points) demonstrated significantly more lesion filling than higher cell density treatments. In addition, all low cell density treated explants demonstrated adhered MSCs to their surface, whereas this was not the case for the high cell density treatment. Although dose-dependent effects have been reported for allogeneic MSCs for the treatment of myocardial infarction in rats [38] and graft versus host disease in mice [39], others have also demonstrated a superior outcome using a lower density of allogeneic MSCs for treatment of injured medial collateral ligaments of rat knees [40] or for the treatment of human knee osteoarthritis [41]. As clump formation did not differ between the cell density groups, the difference was unlikely due to steric obstruction. Hence, more research is warranted to identify the optimal cell density of MSC treatments (rather than the lowest effective dose) such as dose-response studies typical for clinical medicine.

Whereas predifferentiation, loading and seeding density had significant impact on this study's outcome measures, PEMF treatment did not obviously improve any of the evaluated cartilage regeneration parameters in this organ culture study. On the other hand, in the cell culture study an increase in COL II expression was observed in response to PEMF stimulation. Alternative approaches to improve regeneration capacity in explants treated with PEMFs may include: 1) increasing exposure amplitude; 2) increasing exposure duration; 3) increasing number of exposures to a value greater than $1 ; 4)$ exposing MSC-seeded explants directly to PEMFs or; 5) a combination of the above. In fact, Esposito et al. have previously reported induction of chondrogenic differentiation upon PEMF treatment [14] and several in vivo studies have demonstrated beneficial effects of this therapy on clinical symptoms of animals and humans with osteoarthritis $[11,12]$. It has to be mentioned though that these studies applied 6 weeks of daily treatment of the injured joint, whereas our study employed single 10 minute prestimulation of chondrogenically induced MSCs and unstimulated ex vivo cartilage explants. Future research focussing on PEMF-treated cartilage explants might provide more insights into the mechanisms of PEMF stimulation at a tissue level. Such studies might also clarify whether PEMF-treatment evokes cellular cascades of non-cartilage origin.

In conclusion, our results indicate that chondrogenic induction was successful in PBMSCs and resulted in improved outcome with regard to MSC adhesion and integration. PEMF-treatment was able to enhance the expression of COL II in chondrogenically-induced MSCs, which was previously insensitive to induction alone. The application of lower densities of MSCs to explants proved superior to higher MSCs densities, whereas PEMF-treatment exerted no obvious benefit over induction alone in explants. Importantly, immediate physiological loading negatively influenced MSC adhesion to explants, which may have significant implications for the early rehabilitation strategy of patients undergoing MSCs treatment for cartilage regeneration. Future studies will aim to analyse the time-dependent effect of loading of MSC-seeded cartilage explant.

\section{Acknowledgements}

The authors would like to thank Dr. Luk Van Esbroeck of the Euro Meat Group and Chevideco for allowing an optimal and sterile cartilage collection. Sarah Loomans, Christian Puttevils and Delphine Ameye from the Department of Pathology, Bacteriology and Poultry Diseases (Ghent University) as well as Suchandrima Das and Helen Greutert (ETH Zurich) for their technical assistance. We would also like to thank Dr. Jürg Fröhlich and Dr. Christian Beyer of the Department Information Technology and Electrical Engineering of the ETH, 


\section{Cellular Physiology Cell Physiol Biochem 2015;37:651-665 \\ \begin{tabular}{l|l} 
DOI: 10.1159/000430384 & (C) 2015 S. Karger AG, Basel
\end{tabular} \\ and Biochemistry Published online: September 08, $2015 \quad$ www.karger.com/cpb \\ Spaas et al.: Chondrogenic Priming Enhances MSC Adhesion}

Zurich, for supporting the PEMF apparatus used in this study. In addition, the Federal Public Service of Health should be acknowledged for providing GST with a laboratory recognition number (LA1700607), allowing us to perform this study.

This study was supported by a grant (number 130543) to JHS and SYB from the agency for innovation by science and technology Flanders (IWT Vlaanderen). Furthermore, the authors would like to acknowledge GST and the ANACURA group with the sources of private funding, which have provided the basis for this study.

\section{Disclosure Statement}

The author JHS declares competing financial interests as shareholder in Global Stem cell Technology (GST). SYB and JHS are both employed by GST and inventors of several pending patents owned by GST (BE2012/0656; W02014053418A9; W02014053420A1; PCT/EP2013/075782). All other authors declare no conflicts of interests. The content of this manuscript contains a product under development owned by GST.

\section{References}

1 Barbour KE, Helmick CF, Theis KA, Murphy LB, Hootman JM, Brady TJ, Cheng YJ: Prevalence of DoctorDiagnosed Arthritis and Arthritis-Attributable Activity Limitation — United States, 2010-2012. Morbidity and Mortality Weekly Report (MMWR), National Center for Chronic Disease Prevention and Health Promotion, CDC 2013;62:869-873.

2 Murphy L, Schwartz TA, Helmick CG, Renner JB, Tudor G, Koch G, Dragomir A, Kalsbeek WD, Luta G, Jordan JM: Lifetime risk of symptomatic knee osteoarthritis. Arthritis Rheum 2008;59:1207-1213.

3 Hiligsmann M, Cooper C, Arden N, Boers M, Branco JC, Luisa Brandi M, Bruyere O, Guillemin F, Hochberg MC, Hunter DJ, Kanis JA, Kvien TK, Laslop A, Pelletier JP, Pinto D, Reiter-Niesert S, Rizzoli R, Rovati LC, Severens JL, Silverman S, Tsouderos Y, Tugwell P, Reginster JY: Health economics in the field of osteoarthritis: an expert's consensus paper from the European Society for Clinical and Economic Aspects of Osteoporosis and Osteoarthritis (ESCEO). Semin Arthritis Rheum 2013;43:303-313.

4 Caron JP, Genovese RL: Principals and practices of joint disease treatment; in: Ross MW, Dyson S (eds): Diagnosis and management of lameness in the horse. 1st ed. Philadelphia, Saunders, 2003, pp 746-764.

5 Frisbie DD: Future directions in treatment of joint disease in horses. Vet Clin North Am Equine Pract 2005;21:713-724,viii.

6 Jeffcott LB, Rossdale PD, Freestone J, Frank CJ, Towers-Clark PF: An assessment of wastage in thoroughbred racing from conception to 4 years of age. Equine Vet J 1982;14:185-198.

7 Hart BA, Bontrop RE: The relevance of arthritis research in non-human primates. Br J Rheumatol 1998;37:239-242.

8 Charlesworth B, Charlesworth D: Evolution of the Genome; in Ruse M, Travis J (eds): Evolution: The First Four Billion Years. Harvard College, 2009, pp 141-161.

9 Frisbie DD, Cross MW, McIlwraith CW: A comparative study of articular cartilage thickness in the stifle of animal species used in human pre-clinical studies compared to articular cartilage thickness in the human knee. Vet Comp Orthop Traumatol 2006;19:142-146.

10 U.S. Food and Drug Administration: Cellular Products for Joint Surface Repair. Cellular, Tissue, and Gene Therapies Advisory Committee. 2005; Meeting \# 38:27.

11 Boopalan PR, Arumugam S, Livingston A, Mohanty M, Chittaranjan S: Pulsed electromagnetic field therapy results in healing of full thickness articular cartilage defect. Int Orthop 2011;35:143-148.

12 Iannitti T, Fistetto G, Esposito A, Rottigni V, Palmieri B: Pulsed electromagnetic field therapy for management of osteoarthritis-related pain, stiffness and physical function: clinical experience in the elderly. Clin Interv Aging 2013;8:1289-1293.

13 Trock DH, Bollet AJ, Markoll R: The effect of pulsed electromagnetic fields in the treatment of osteoarthritis of the knee and cervical spine. Report of randomized, double blind, placebo controlled trials. J Rheumatol 1994;21:1903-1911. 


\section{Cellular Physiology Cell Physiol Biochem 2015;37:651-665 \begin{tabular}{l|l|l} 
DOI: 10.1159/000430384 & (C) 2015 S. Karger AG, Basel
\end{tabular} and Biochemistry Published online: September 08, $2015 \quad$ www.karger.com/cpb \\ Spaas et al.: Chondrogenic Priming Enhances MSC Adhesion}

14 Esposito M, Lucariello A, Costanzo C, Fiumarella A, Giannini A, Riccardi G, Riccio I: Differentiation of human umbilical cord-derived mesenchymal stem cells, WJ-MSCs, into chondrogenic cells in the presence of pulsed electromagnetic fields. In Vivo 2013;27:495-500.

15 Chen CH, Lin YS, Fu YC, Wang CK, Wu SC, Wang GJ, Eswaramoorthy R, Wang YH, Wang CZ, Wang YH, Lin SY, Chang JK, Ho ML: Electromagnetic fields enhance chondrogenesis of human adipose-derived stem cells in a chondrogenic microenvironment in vitro. J Appl Physiol (1985) 2013;114:647-655.

16 Frisbie DD, Kisiday JD, Kawcak CE, Werpy NM, MccIlwraith CW: Evaluation of adipose-derived stromal vascular fraction or bone marrow-derived mesenchymal stem cells for treatment of osteoarthritis. J Orthop Res 2009;27:1675-1680.

17 Mcllwraith CW, Frisbie DD, Rodkey WG, Kisiday JD, Werpy NM, Kawcak CE, Steadman JR: Evaluation of intra-articular mesenchymal stem cells to augment healing of microfractured chondral defects. Arthroscopy 2011;27:1552-1561.

18 Wilke MM, Nydam DV, Nixon AJ: Enhanced early chondrogenesis in articular defects following arthroscopic mesenchymal stem cell implantation in an equine model. J Orthop Res 2007;25:913-925.

19 Broeckx S, Zimmerman M, Crocetti S, Suls M, Marien T, Ferguson SJ, Chiers K, Duchateau L, Fraco-Obregon A, Wuertz K, Spaas JH: Regenerative therapies for equine degenerative joint disease: a preliminary study. PLoS One. 2014;9:e85917.

20 Broeckx S, Suls M, Beerts C, Vandenberghe A, Seys B, Wuertz-Kozak K, Duchateau L, Spaas JH: Allogenic mesenchymal stem cells as a treatment for equine degenerative joint disease: a pilot study. Curr Stem Cell Res Ther 2014;9:497-503.

21 Chen K, Man C, Zhang B, Hu J, Zhu SS: Effect of in vitro chondrogenic differentiation of autologous mesenchymal stem cells on cartilage and subchondral cancellous bone repair in osteoarthritis of temporomandibular joint. Int J Oral Maxillofac Surg 2013;42:240-248.

22 Broeckx S, Zimmerman M, Aerts D, Seys B, Suls M, Mariën T, Spaas JH: Tenogenesis of equine peripheral blood-derived mesenchymal stem cells: in vitro versus in vivo. J Tissue Sci Eng 2012;S11-001:1-6.

23 Spaas JH, Schauwer CD, Cornillie P, Meyer E, Van Soom A, Van de Walle GR: Culture and characterisation of equine peripheral blood mesenchymal stromal cells. Vet J 2013;195:107-113.

24 Crocetti S, Beyer C, Schade G, Egli M, Frohlich J, Franco-Obregon A: Low intensity and frequency pulsed electromagnetic fields selectively impair breast cancer cell viability. PLoS One 2013;8:e72944.

25 Krupkova O, Sekiguchi M, Klasen J, Hausmann O, Konno S, Ferguson SJ, Wuertz-Kozak K: Epigallocatechin 3-gallate suppresses interleukin-1beta-induced inflammatory responses in intervertebral disc cells in vitro and reduces radiculopathic pain in rats. Eur Cell Mater 2014;28:372-386.

26 Dudli S, Haschtmann D, Ferguson SJ: Persistent degenerative changes in the intervertebral disc after burst fracture in an in vitro model mimicking physiological post-traumatic conditions. Eur Spine J 2014.

27 Hemmrich K, Meersch M, von Heimburg D, Pallua N: Applicability of the dyes CFSE, CM-DiI and PKH26 for tracking of human preadipocytes to evaluate adipose tissue engineering. Cells Tissues Organs 2006;184:117-127.

28 Shuai H, Shi C, Lan J, Chen D, Luo X: Double labelling of human umbilical cord mesenchymal stem cells with Gd-DTPA and PKH26 and the influence on biological characteristics of hUCMSCs. Int J Exp Pathol 2015.

29 Qu B, Xin GR, Zhao LX, Xing H, Lian LY, Jiang HY, Tong JZ, Wang BB, Jin SZ: Testing stem cell therapy in a rat model of inflammatory bowel disease: role of bone marrow stem cells and stem cell factor in mucosal regeneration. PLoS One 2014;9:e107891.

30 Progatzky F, Dallman MJ, Lo Celso C: From seeing to believing: labelling strategies for in vivo cell-tracking experiments. Interface focus 2013;3:20130001.

31 Weinand C, Johnson TS, Gill TJ: In vitro and in vivo cell tracking of chondrocytes of different origin by fluorescent PKH 26 and CMFDA. J Biomed Sci Eng 2008;1:163-169.

32 Ude CC, Sulaiman SB, Min-Hwei N, Hui-Cheng C, Ahmad J, Yahaya NM, Saim AB, Idrus RB: Cartilage regeneration by chondrogenic induced adult stem cells in osteoarthritic sheep model. PLoS One 2014;9:e98770.

33 Vinardell T, Thorpe SD, Buckley CT, Kelly DJ: Chondrogenesis and integration of mesenchymal stem cells within an in vitro cartilage defect repair model. Ann Biomed Eng 2009;37:2556-2565.

34 Fowlie J, Arnoczky S, Lavagnino M, Maerz T, Stick J: Resection of Grade III cranial horn tears of the equine medial meniscus alter the contact forces on medial tibial condyle at full extension: an in-vitro cadaveric study. Vet Surg 2011;40:957-965. 


\section{Cellular Physiology Cell Physiol Biochem 2015;37:651-665 \begin{tabular}{l|l|l|}
\hline DOI: 10.1159/000430384 & C 2015 S. Karger AG, Basel
\end{tabular} \\ Spaas et al.: Chondrogenic Priming Enhances MSC Adhesion}

35 Murray RC, Vedi S, Birch HL, Lakhani KH, Goodship AE: Subchondral bone thickness, hardness and remodelling are influenced by short-term exercise in a site-specific manner. J Orthop Res 2001;19:10351042.

36 Chu CR, Szczodry M, Bruno S: Animal models for cartilage regeneration and repair. Tissue Eng Part B, Reviews 2010;16:105-115.

37 Ahern BJ, Parvizi J, Boston R, Schaer TP: Preclinical animal models in single site cartilage defect testing: a systematic review. Osteoarthr Cartil 2009;17:705-713.

38 Richardson JD, Bertaso AG, Psaltis PJ, Frost L, Carbone A, Paton S, Nelson AJ, Wong DT, Worthley MI, Gronthos S, Zannettino AC, Worthley SG: Impact of timing and dose of mesenchymal stromal cell therapy in a preclinical model of acute myocardial infarction. J Card Fail 2013;19:342-353.

39 Joo SY, Cho KA, Jung YJ, Kim HS, Park SY, Choi YB, Hong KM, Woo SY, Seoh JY, Cho SJ, Ryu KH: Mesenchymal stromal cells inhibit graft-versus-host disease of mice in a dose-dependent manner. Cytotherapy 2010;12:361-370.

40 Saether EE, Chamberlain CS, Leiferman EM, Kondratko-Mittnacht JR, Li WJ, Brickson SL, Vanderby R: Enhanced medial collateral ligament healing using mesenchymal stem cells: dosage effects on cellular response and cytokine profile. Stem Cell Rev 2014;10:86-96.

41 Vangsness CT, Farr J, Boyd J, Dellaero DT, Mills CR, LeRoux-Williams M: Adult human mesenchymal stem cells delivered via intra-articular injection to the knee following partial medial meniscectomy: a randomized, double-blind, controlled study. J Bone Joint Surg Am 2014;96:90-98. 